\title{
Mitral Regurgitation in Hypertrophic Cardiomyopathy: A Narrative Review of Mechanism and Current Management
}

\author{
Ashraf M Anwar, MD, PhD, FESC ${ }^{1,2^{*}}$ \\ ${ }^{1}$ Department of Cardiology, King Fahad Armed Forces Hospital, Jeddah, Saudi Arabia \\ ${ }^{2}$ Department of Cardiology, Faculty of Medicine, Al-Azhar University, Cairo, Egypt
}

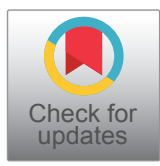

*Corresponding author: Ashraf M Anwar; MD, PhD, FESC, Cardiology Department, King Fahd Armed Forces Hospital, P.O. Box: 9862, Jeddah 21159, Saudi Arabia, Fax: +966-2-6651868

\begin{abstract}
Significant mitral regurgitation (MR) is frequent in patients with hypertrophic cardiomyopathy (HCM) either obstructive or non-obstructive. Many studies described that significant MR is associated with poor long-term outcome of HCM patients, which indicate the importance of an adequate MR assessment including detailed evaluation of the mitral valve (MV) anatomy. Mitral malformations have been identified in $\mathrm{HCM}$ at all levels. They occur in the MV leaflets with a wide array of malformations of the papillary muscles and chordae. Complete understanding the mechanism of MR and accurate quantification can guide towards the selection of therapeutic modality. Multimodality imaging helps describe mitral abnormalities in HCM. Transthoracic and trans-esophageal echocardiographies as well as magnetic resonance imaging emerges are the most important imaging modalities for precise diagnosis, risk stratification and treatment strategy. This review will focus on the abnormalities of the MV apparatus for understanding the mechanism MR in HCM with a discussion of the value of multimodality imaging approach to diagnosis and quantification. Also, the review will describe the standard management and new optional techniques with the evidence from the published data.
\end{abstract}

\section{Keywords}

Hypertrophic cardiomyopathy, Mitral regurgitation, Narrative review

\section{List of Abbreviations}

ASA: Alcohol Septal Ablation; CMR: Cardiac Magnetic Resonance Imaging; EF: Ejection Fraction; HCM: Hypertrophic Cardiomyopathy; LV: Left Ventricle; LVOT: Left Ventricular Outflow Tract; LVOTO: Left Ventricular Outflow Tract Obstruction; MR: Mitral Regurgitation; MV: Mitral Valve; RFA: Radiofrequency Ablation; TTE: Transthoracic Echocardiography; TEE: Trans-esophageal Echocardiography; RV: Right Ventricle; SAM: Systolic Anterior Motion; 2DE: 2-Dimensional Echocardiography; 3DE: 3-Dimensional Echocardiography

\section{Introduction}

Up to $70 \%$ of patients with hypertrophic cardiomyopathy (HCM) exhibit left ventricular outflow tract obstruction (LVOTO) [1]. LVOTO is frequently associated with significant mitral regurgitation (MR) because of systolic anterior motion (SAM) of mitral leaflets (SAM- dependent MR). Significant MR may occur in up to $10 \%$ to $20 \%$ of HCM patients without SAM (SAM-independent MR) and is due to intrinsic mitral valve (MV) abnormalities [2]. LVOTO together with SAM-dependent MR leads to progressive deterioration of clinical status and poor long-term outcome. Patients with $\mathrm{HCM}$ and significant MR had more frequent chest pain, syncope and NYHA $\geq$ II functional class. MR is one of the factors responsible for left atrial enlargement and is an important cause of atrial arrhythmias, particularly atrial fibrillation [3]. Significant symptomatic LVOTO requires septal reduction therapy, which may vary depending on the mechanisms of LVOTO, MV disease status and alterations in papillary muscles $[3,4]$. The coexistence of MR may influence the decision between intervention and conservative treatment. Some centers consider septal reduction therapy is enough in $\mathrm{HCM}$ patients with significant LVOTO if they have moderateto-severe SAM-dependent MR. Some reports showed that concomitant MV surgery is associated with sustained improvement of SAM, MR, LVOTO, reoperation, and functional status [3].

\section{Mechanism of MR}

SAM-dependant MR: In most patients with obstructive HCM, the MR that is dependent on SAM 
peaks in mid and late systole. SAM is initiated by rapid LV ejection through an LVOT narrowed by ventricular septal hypertrophy and anterior displacement of the $\mathrm{MV}$ apparatus. This result in production of drag forces, which draw both the MV leaflets and chordae towards the interventricular septum. Anterior displacement of the papillary muscles shifts the MV leaflets anteriorly toward the LVOT and leads to chordal and leaflet laxity. As drag forces generated by the LV pull the anteriorly displaced and elongated leaflets into the outflow tract in early systole, the distal one half to one third of the leaflets form an angle anteriorly into the LVOT, creating a "funnel" composed of both leaflets. Narrowing of the LVOT leads to dynamic pressure gradients and in many patients, MR severity is dynamic and is affected by the same factors that influence the severity of LVOTO $[5,6]$.

Because the anterior leaflet motion towards the LVOT is greater than that of the posterior leaflet during SAM, the coaptation point between the anterior and posterior leaflets is typically eccentric and interleaflet gap is created. This results in a typically posteriorly directed jet of $M R$ forming a right angle with the turbulent LVOT flow, and occurs in mid to late systole. The degree of MR relates to the extent of mismatch of anterior to posterior leaflet length and the decreased mobility of the posterior leaflet to move anteriorly which can be significant (moderate or greater depending on the extent of the gap) $[7,8]$.

Intrensic MR (SAM-independent): Significant MR due to MV abnormalities may occur in up to $10 \%$ to $20 \%$ of patients with $\mathrm{HCM}$ who carry a genetic mutation for HCM but have not manifested septal hypertrophy or LVOTO [9]. SAM-independent MR is usually directed anteriorly or centrally into the left atrium and is usually holosystolic. Intrinsic MV disease is often obscured by the uniform presence of dynamic MR, but presence of MR without SAM or presence of an anteriorly directed jet is strong evidence for intrinsic MV disease [10].

\section{MV abnormalities}

The true prevalence of mitral abnormalities in HCM is difficult to assess, as many abnormalities are poorly appreciated and often not documented. It is known that up to $59 \%$ of patients with HCM have at least one abnormality of the MV apparatus as a direct effect of genetic mutations [11]. MV abnormalities may be present in any component of the mitral apparatus (Leaflets, subvalvular apparatus and annulus) and thus numerous abnormalities have been described with wide variability among patients with both obstructive and non-obstructive types of HCM.

\section{Leaflet abnormalities:}

a. Excessive leaflet elongation and increased leaflet area are present in about $50 \%$ of patients and can involve one or both MV leaflets. Leaflet elongation is a unique finding of HCM. However, the leaflet size is more related to chamber geometry and hypertrophy pattern rather than genetic factors within overt HCM [12]. The anterior leaflet averages $34 \mathrm{~mm}$ in HCM versus $24 \mathrm{~mm}$ in normal hearts. MV leaflets are longer in patients with obstructive HCM than with non-obstructive HCM. An increased ratio of anterior leaflet length to LVOT diameter is associated with resting and provocable obstruction [13]. A posterior leaflet of $>14 \mathrm{~mm}$ was observed in carriers of a morbid HCM mutation, but without hypertrophy or obstruction which suggests sarcomere gene mutation rather than leaflet stress or ventricular remodeling [9]. The pathological series reported associated thickening of the elongated leaflet(s) as seen in myxomatous disease. Whether leaflet elongation is an early form or variant manifestation of myxomatous MV disease is unknown [14].

b. Restrictive leaflets with valve calcification and thickening are the most common form of intrinsic MV disease identified. The mechanism of valve thickening and calcification is unknown, but the traumatic fibrosis from repetitive septal contact may result in retraction, and MR. In patients with restricted, calcified, and thickening leaflets with resting SAM, it is difficult to determine whether the MR is caused solely by SAM of the MV or is related in part to intrinsic MV disease [15].

c. MV prolapse: The frequency of coexisting MV prolapse with HCM and the potential clinical significance of such an association are not studied well. MV prolapse was observed in $44 \%$ of the 45 patients with HCM [16] while in a larger group (528 patients), it was identified in only 3\% [17].

Subvalvular abnormalities: Subvalvular abnormalities are described in more than $50 \%$ of $\mathrm{HCM}$ patients and include [18-20]:

a. Hypertrophy of papillary muscle heads, with or without septal or posterior wall hypertrophy, which can cause mid cavity obstruction.

b. Increased number of papillary muscles; 3-4 papillary muscle heads occur in more than $50 \%$ of HCM patients.

c. Anterior and apically displaced papillary muscle that shifts the MV leaflets anteriorly toward the LV outflow and leads to chordal and leaflet laxity.

d. Anomalous insertion of one or both heads of the anterolateral papillary muscle directly to the ventricular aspect of the tip, mid or base of anterior MV leaflet. It was recognized in up to $13 \%$ of patients with $\mathrm{HCM}$ and can contribute to LVOTO.

e. Fibrotic and retracted secondary chordae can 
result in tethering and tenting of the anterior $\mathrm{MV}$ leaflet toward the LVOT flow stream.

f. Chordal rupture is a common complication of repetitive stress on the mitral apparatus and involves essentially the posterior MV leaflet that leads to severe MR. Myxomatous degeneration may be the substrate for rupture in these patients. Chordal rupture should be considered in the differential diagnosis of acutely deteriorated $\mathrm{MR}$ in patients with HCM.

MV annulus abnormalities: Changes in size, shape, and angulation of the MV annulus in HCM are less appreciated. It was shown that mital annulus area is enlarged in both obstructive and non-obstructive HCM patients compared to normal as detected by 3D echocardiography and MRI. In both obstructive and non-obstructive HCM types, annulus dilatation occurs to a certain limit beyond which more stretch is prevented by the hyperactive muscular part even with significant MR [21,22]. Given the stressful hemodynamic loading conditions in HCM, marked mitral annular calcification may be seen [23].

\section{Approach to diagnose MR in HCM}

Echocardiography: Echocardiography is the most informative technique aids in the diagnosis, risk stratification, treatment selection and follow up of HCM patients. Comprehensive trans-thoracic echocardiography (TTE) helps to understand the mechanism, characterize and assess severity of MR in patients with HCM. Transesophageal echocardiography (TEE) has strength in clarifying anatomy and mechanism of $M R$, with better 2D and 3D imaging of the annulus and leaflets. Intraoperative TEE during structural intervention allows real-time assessment of preoperative anatomy, hemodynamics, and mechanism of MR. Compared with preoperative TTE, intraoperative
TEE identifies new findings in about $20 \%$ of patients with $\mathrm{HCM}$ that can significantly influence operative planning and outcome [24]. The echocardiographic assessment should include full information about the following:

- Quantification of MR: MR severity can be assessed by vena contracta and/or measurement of effective orifice area when it is feasible by 2D-TTE or 2D-TEE. 3D echocardiography enables the precise location of the regurgitant jet origin and direction and direct assessment of the size, shape and symmetry of the vena contracta area. Calculation of regurgitant volume and fraction is liable for error especially with SAMdependent MR due to LVOT morphology. All indicators of MR severity should be considered to obtain accurate MR quantification [25].

- Differentiation between SAM-dependent and SAM- independent types of MR using jet direction. An anteriorly directed jet suggests an intrinsic $M V$ abnormality. Laterally and posteriorly directed jet predominates during mid and late systole in obstructive HCM and correlates highly with SAM (positive predictive value $94.9 \%$ on TTE and $97.1 \%$ on intraoperative TEE) (Figure 1). Isolated posterior jets detected by TTE had sensitivity and specificity of $65.1 \%$ and $67.9 \%$ for identifying SAM-mediated MR while by TEE, the sensitivity and specificity was $59.5 \%$ and $82.1 \%$ respectively [26].

- Morphologic assessment of MV: It is essential to obtain a careful and detailed anatomical description of MV apparatus [27-29].

1. Length of both MV leaflets in diastole from the annulus to its chordal insertion and their ratio.

2. Mobility of both leaflets: Measurement of the angle between the posterior LV wall and the basal part of the posterior leaflet (angle- $\alpha$ ) and the angle between the basal and distal parts of

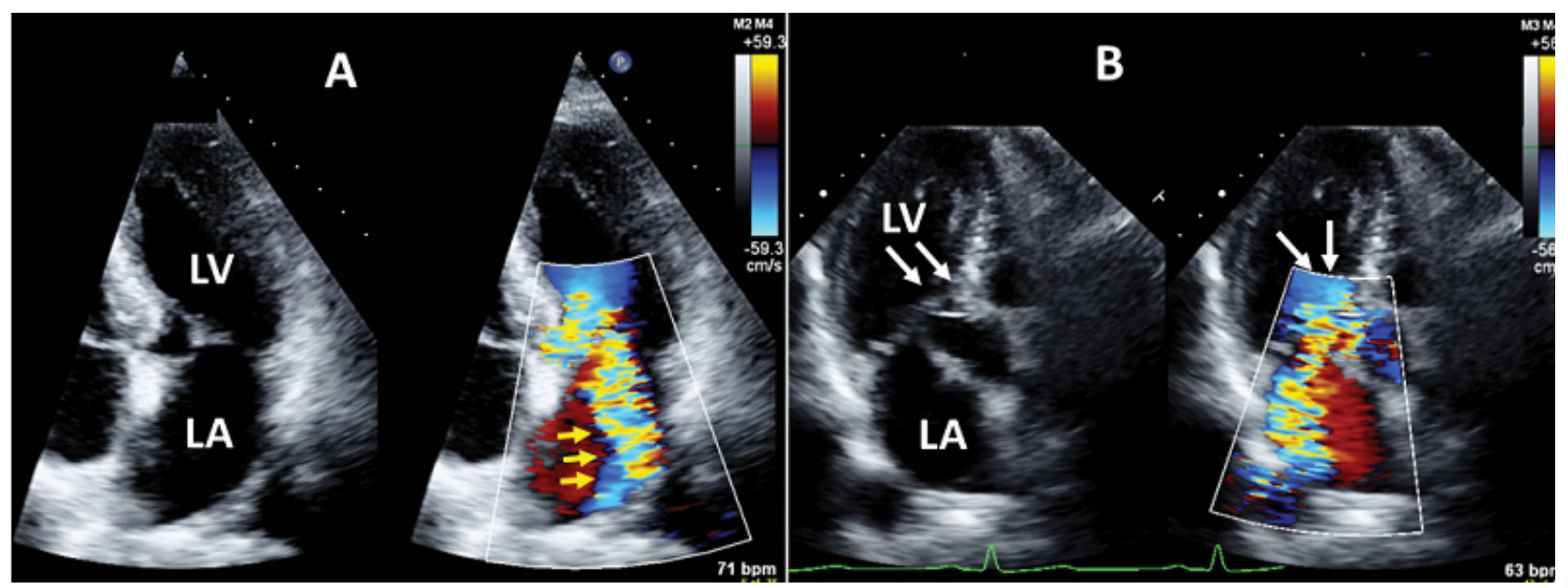

Figure 1: 2D transthoracic echocardiography showed SAM-dependent significant MR; A) Apical 4-chamber view with color Doppler showed significant eccentric MR (Yellow arrows) and B) Apical long axis view showed narrowed LVOT by SAM (white arrow) with color acceleration. 
the posterior leaflet (angle- $\beta$ ) can be used as indicator for posterior leaflet mobility. The larger the angles the greater the mobility.

3. Leaflet coaptation length, depth and gap should be measured at end-diastole and can be used in the perioperative evaluation of the competency of the MV.

4. Chordal thickening, elongation and degree of systolic buckling with interventricular septum should be assessed with a careful looking for aberrant chordae, partial or complete rupture

5. Number of papillary muscles (duplication/bifidity), apical/anterior displacement, hypertrophy and/ or anomalous papillary muscle attached directly into the MV should be considered.

6. MV annulus diameter (anterior-posterior and inter-commissural) can be measured using 2D-TTE and/or TEE. 3D echocardiography enables measurement of annulus circumference and area.

Cardiac magnetic resonance (CMR): CMR helps to characterize MV and papillary muscle size, location and attachment. These findings are of potential value in understanding the mechanism of MR and planning surgery for the treatment of dynamic obstruction. CMR has superior spatial resolution and is not limited by imaging windows, therefore it should be considered in patients with suboptimal visualization of the MV or papillary muscles by echocardiography, or when the TEE is declined or contraindicated. Quantification of MR severity is performed more commonly by echocardiography. CMR quantifies MR as the difference between LV stroke volume and aortic or pulmonary forward flow $[9,30]$.

Cardiac computerized tomography: Computerized tomography has been shown to reliably measure leaflet lengths, estimate the degree of annulus calcification, and assess papillary muscle abnormalities (number, position and extent of hypertrophy). However, the application of the technique for the assessment of MR in patients with HCM has not been established [31].

Nuclear imaging: Using nuclear imaging, it is possible to quantify the severity but not the mechanisms of MR as the difference between LV and RV stroke volumes.

\section{Treatment of MR associated obstructive HCM}

The treatment strategies of $\mathrm{HCM}$ are based upon observational data and clinical experience. The approach of treatment has largely been limited to symptomatic patient with obstructive HCM. For asymptomatic patients, close clinical follow-up is the preferred approach because there is no evidence for any therapeutic modality that it alters the natural history of the disease prior to the onset of symptoms.

Medical treatment: There have been no large randomized trials of pharmacologic therapies in HCM. Current medical therapy of negative inotropic and chronotropic drugs including B-blockers, Ca channel blockers, and Disopyramide have a modest effect on LVOT gradient and should be avoided in severely symptomatic patients with severe resting LVOT gradient $>80 \mathrm{mmHg}$. Using these drugs even with combination failed to address the core pathophysiology of HCM without affecting its natural history [32]. Mavacamten is a novel cardiac myosin inhibitor targeting reduction of cardiac myocytes contractility by inhibiting ATPase activity of myosin. EXPLORER-HCM trial conducted 251 patients with obstructive HCM treated with Mavacamten on top of their previous B-blocker or Ca channel blocker therapy. Nearly three-fourths of the patients on Mavacamten showed significant LVOT gradient reduction below the guideline-defined threshold for invasive therapy (post-exercise LVOT peak gradient < $50 \mathrm{mmHg}$ ). Nearly $60 \%$ experienced a complete relief from obstruction (LVOT gradient < $30 \mathrm{mmHg}$ ) and about $30 \%$ reached a complete relief from both symptoms and obstruction (NYHA class I and exercise-induced gradient $<30 \mathrm{mmHg}$ ) [33]. Using CMR, EXPLORER-HCM sub-study showed that Mavacamten induced reverse cardiac remodeling, reducing $L V$ mass index and maximum LV wall thickness, great reduction in LV volume index and a decrease in LV filling pressure. Compared to placebo, Mavacamten achieved restoration of MV motion and resolved SAM in $80.9 \%$ vs. $34 \%$ of patients with obstructive $\mathrm{HCM}$ and significant reduction of $\mathrm{MR}$ in $9 \%$ vs. $0 \%$ [34]. In non-obstructive HCM, MAVERICK-HCM trial (phase II) showed treatment with Mavacamten was associated significant reduction in cardiac biomarker which indicated improvement of myocardial wall stress [35].

Surgical treatment: There is controversy regarding the optimal surgical strategy whether to perform septal myectomy alone or concomitant mitral operation is needed?. Septal myectomy/extended septal myectomy is currently the gold standard for HCM with moderate to severe LVOTO and is class I indication for severely symptomatic patients refractory to optimal medical management. The surgical approach involves obtaining access to the interventricular septum via transaortic, transapical or transmitral approaches, and excising a portion of the hypertrophied myocardium to relieve the LVOTO. Adequate subaortic septal myectomy eliminates or ameliorates SAM of the MV, thus improving SAMdependent MR and reducing left atrial size in most patients if there is no intrinsic MV disease [2]. Data from a large series from five high-volume centers experienced in septal myectomy over the 15-year period have demonstrated very low operative mortality $(0.4 \%)$ in 3700 isolated septal myectomy operations [36]. Concomitant MV surgery performed at the same time as myectomy have increasingly been applied in patients with mitral and papillary muscle anomalies 
as identified on preoperative echocardiography or by the direct surgical inspection. Some reports showed associated sustained improvement of SAM, MR, LVOTO, re-operation, and functional status. Some showed increased length of stay and increased hospital mortality $[37,38]$. No randomized controlled trial comparing the results of isolated myectomy versus myectomy plus MV repair. Only surgeon experience can judge that isolated myectomy will be inadequate to resolve the SAM. Additionally, pre-operative and intraoperative TTE and TEE can help in the detailed assessment of the complex anatomy of the LVOT, septal thickness, MV leaflet length, and papillary muscles abnormalities [39]. Similar to the recommendations regarding surgery for patients with asymptomatic MV disease, earlier surgery in patients with HCM should be limited to those comprehensive $\mathrm{HCM}$ centers with documented evidence of the highest success rates and lowest complication rates (i.e., durable success is $>90 \%$ with an expected mortality rate $<1 \%$ ) [32]. Many concomitant MV surgical techniques were advocated and the selection between them will be individualized according to the great diversity of mitral abnormalities [40]. The most common techniques are:

1. Leaflet retention plasty [41-44]: Surgical restoration of the $\mathrm{MV}$ anatomy through modifying the anatomic alterations of MV leaflet(s) such as increased leaflet area, length and laxity. According to the echocardiographic data and surgical visualization of the $\mathrm{MV}$ apparatus, one or more of the following techniques will be selected:

- Leaflet resection for an unsupported scallop of the posterior leaflet and artificial chordae or plication for an unsupported scallop of the anterior leaflet with selective use of a flexible posterior annuloplasty band,

- Complete resection and replacement of all anterior leaflet chordae with the loop technique,
- Complete excision of secondary chordae of the anterior leaflet,

- Triangular resection of the anterior leaflet,

- Anterior leaflet extension with a pericardial patch,

- Sliding plasty of the posterior leaflet,

- Resection, Release and debulking of the anteriorly displaced papillary muscle.

2. Edge to edge repair: Repair of MV leaflets through placement of Alfieri stitchin addition to myectomy has also been used effectively for reduction of SAM and improvement in MR. The echocardiographic studies reported good short-term results without recurrence of significant MR during the median follow-up period (6.6 years) [45]. The edge-to-edge repair provides a simple, quick, and effective solution by abolishing the residual LVOT gradient and SAM-dependent MR when septal thickness is inadequate to allow a safe and effective myectomy [46].

3. MV replacement: The studies compared the results of $\mathrm{MV}$ repair and $\mathrm{MV}$ replacement showed that the MV repair had better overall survival $(87.2 \pm 4.9 \%$ and 96.7 $\pm 3.3 \% ; \mathrm{P}=0.034)$ and a lower rate of thromboembolic events $(91.2 \pm 4.2 \%$ and $100 \% ; P=0.026)$ than $M V$ replacement at 2-year follow up [2]. Every effort should be made to perform MV repair as the late survival also is superior with MV repair than with replacement (10year survival: $80.0 \%$ vs. $55.2 \% ; p=0.002$ ) [47]. $M V$ replacement alone can be a successful approach in cases unsuitable for repair.granular calcification, anterior MV leaflet fibrosis or when used alone in those patients with thinner septum unsuitable for myectomy [48]. It should be noted that $\mathrm{MV}$ replacement with a mechanical valve is the compelled strategy and the high-profile biological prosthesis is not a suitable strategy, given the additional risk of LVOT obstruction [49] (Figure 2).
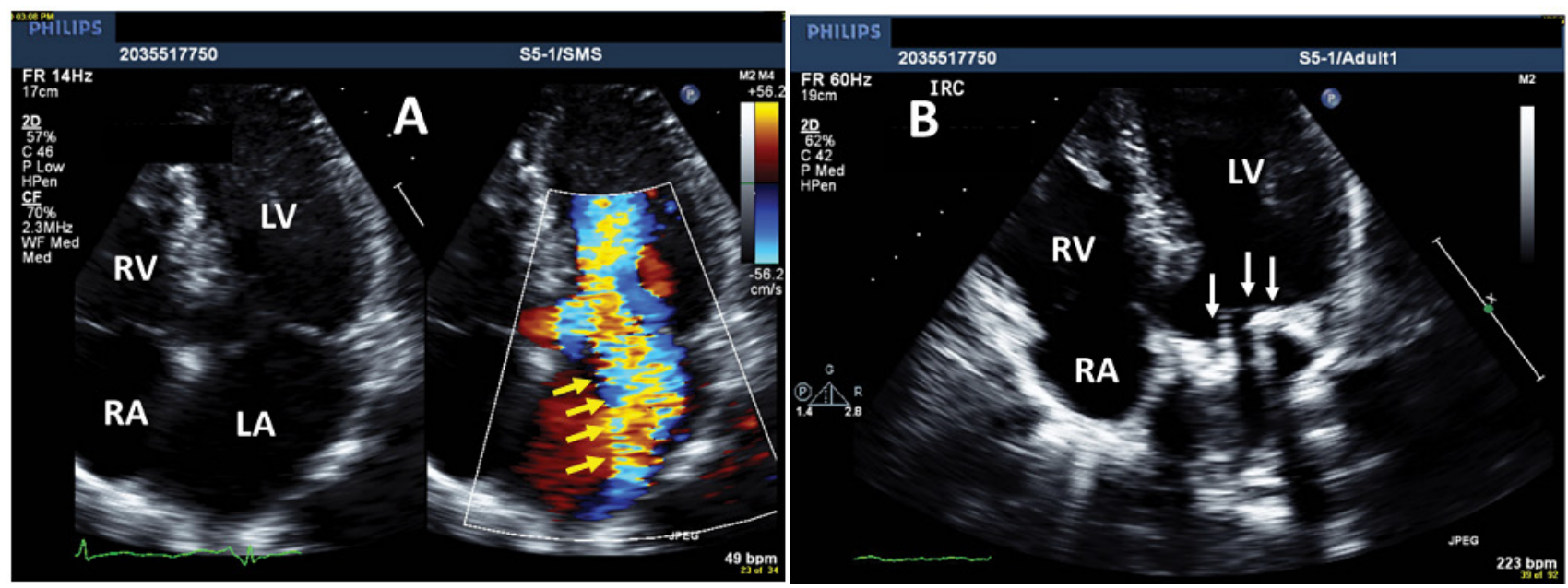

Figure 2: 4-chamber view of 2D-TTE obtained in a patient with obstructive HCM had severe MR; A) Color Doppler showed the eccentric jet of MR (yellow arrows); B) Same 4-chamber view without color after treatment by surgical myectomy and MV replacement with mechanical prosthesis (white arrows). 


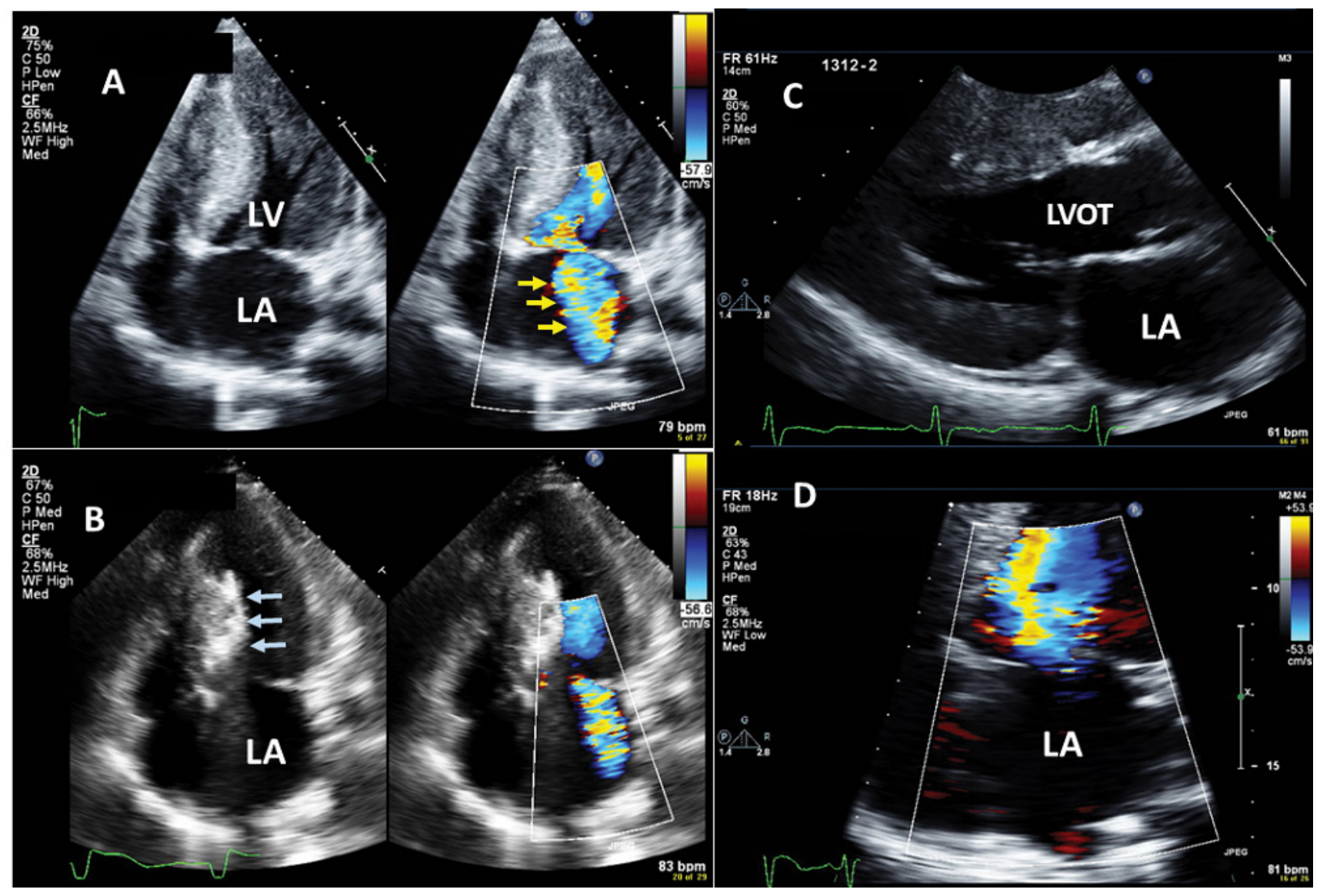

Figure 3: Example of patient with obstructive HCM and severe MR treated by Alcohol septal ablation; A) 4-chamber view showed the MR jet (yellow arrows) before septal ablation; B) 4-chamber view during septal ablation (blue arrows pointed towards the area of ablation); C) parasternal long axis after septal ablation showed relief of LVOT obstruction, and D) 4-chamber view showed no residual MR after septal ablation.

\section{Catheter-based therapies}

1. Alcohol Septal Ablation (ASA): The procedure consists of selective injection of 1.5 to $2.5 \mathrm{~mL}$ absolute alcohol into the major septal branch of the anterior descending artery through the cardiac catheterization. It will cause septal infarction (10\% of the LV wall, $30 \%$ of the septum, on average), with the formation of scarring and septal reduction (Figure 3). Many investigators reported that ASA is a safe procedure with ongoing symptomatic improvement and excellent long-term survival. Liebregts, et al. [50] study included 1,197 patients who underwent ASA at seven centers in four European countries with mean follow-up period of $5.4 \pm 4.2$ years. The annual mortality rates of young, middle-age, and older patients were $1 \%, 2 \%$, and $5 \%$, respectively. The second-largest long-term study included 952 German patients with a minimum 6-year follow-up. The estimated 5-, 10-, and 15-year survival rates were $95.8 \%, 88.3 \%$, and $79.7 \%$, respectively. Estimated survival free of cardiac events at 5, 10 and 15 years was $98.9 \%, 97.0 \%$, and $96.5 \%$ respectively [51]. Alcohol septal ablation has become an alternative to surgical myectomy in select patients, such as those with advanced age, significant comorbidities, or who oppose open chest surgery. The procedure requires appropriate coronary anatomy and should be avoided in patients with high resting gradient ( $\geq 100 \mathrm{mmHg}$ ) and septal thickness $<16$ $\mathrm{mm}$ or $\geq 30 \mathrm{~mm}$ [52]. The decision between ASA and surgical myectomy is influenced by multiple variables including patient comorbidities as well as the experience of the performing providers.

2. MV repair: Transcatheter MV plication is a potential therapeutic option in symptomatic HCM patients who are not surgical candidates and/or do not have optimal coronary anatomy for ASA. The technique offers several potential advantages as it is less invasive, does not create a potentially arrhythmogenic ablation scar requiring a permanent pacemaker, and it targets the MV directly, that causes the LVOTO. Several small studies have investigated transcatheter MV plication (MitraClip) to reduce SAM and LVOT obstruction (Figure 4). Sorajja, et al. [53] published the first experience in the world with placement of a single MitraClip in 5 patients. Follow-up echocardiography after $15 \pm 4$ months demonstrated eliminated SAM and markedly decreased the intraoperative LVOTO and MR. 

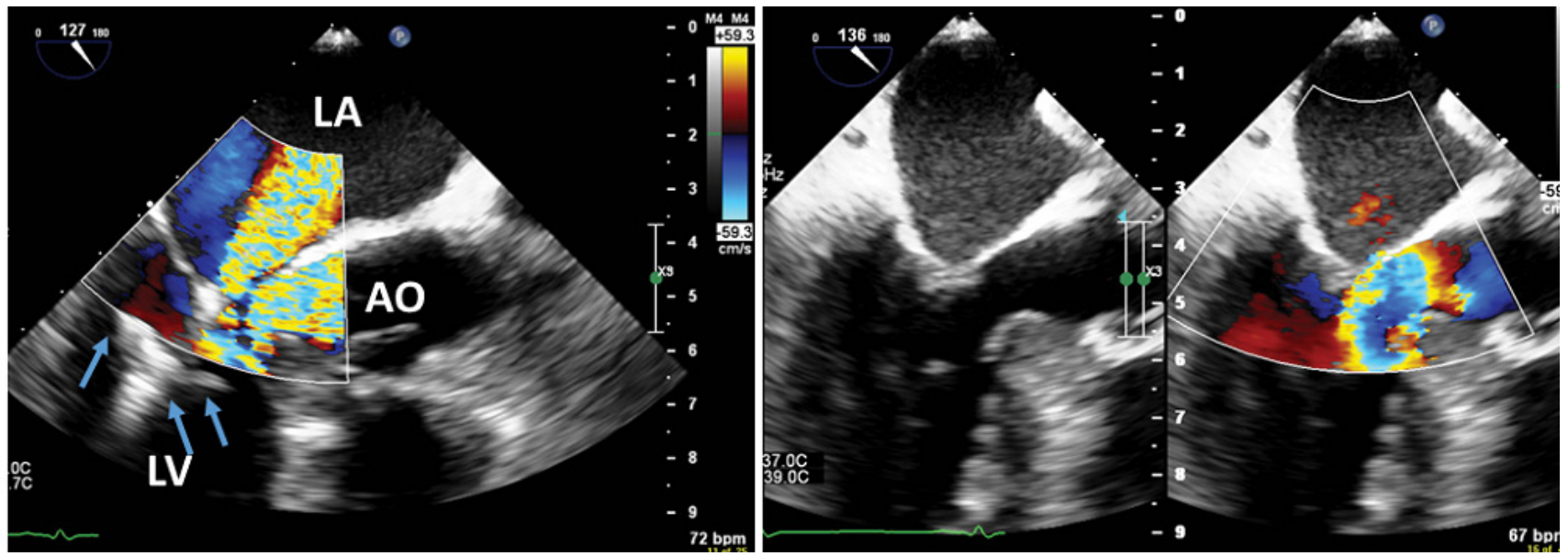

Figure 4: Transesophageal echocardiography showed significant MR in patients with obstructive HCM; A) during the procedure of trans-catheter MV repair using Mitral Clip device (blue arrows) and B) after the clip deployment with reduced color acceleration across LVOT and complete resolution of MR.

Although these studies were limited by size and adequate follow-up. Thomas, et al. [54] collected data from 4 separate studies on the use of the MitraClip on 15 symptomatic HCM patients with SAM. All patients had a resolution of SAM, a reduction in MR, and a reduction in the LVOT gradient from a mean of $75.8 \pm 39.7$ to $11.0 \pm$ $5.6 \mathrm{mmHg}$. More data are needed to determine the long-term efficacy of MitraClip for HCM and whether subsequent interventions with repeat clip, ASA, or myectomy are needed. Future devices may offer additional opportunities to address MV abnormalities in HCM [55].

3. Radiofrequency ablation (RFA): RFA is both minimally invasive and independent of coronary anatomy technique where the energy is is applied to the endocardial surface of the basal interventricular septum from a retrograde aortic or trans-septal approach for septal reduction using electro-anatomic or echocardiographic guidance. Several small series reported modest septal reduction (1-2 $\mathrm{mm}$ compared to $\sim 5-6 \mathrm{~mm}$ with ASA), likely due to the limited subendocardial delivery of energy and lesion formation [56]. Percutaneous intramyocardial septal RFA was reported in a series of 15 patients using TTE to guide transapical placement of an intraseptal RF electrode that delivers energy to the core of the hypertrophic segment. Septal reduction of $11 \mathrm{~mm}$ was achieved with resolution of LVOT obstruction, improvements in NYHA class, and reduced serum NT-proBNP at 6 months. Importantly, there was no conduction defect, likely due to selective delivery of RF energy in the deep septum away from the subendocardial His-Purkinje fibers [57]. Collective data from eight studies with 91 patients (mean follow up 11.6 months) showed that RFA might become a promising invasive treatment for patients with obstructive HCM who are still symptomatic despite receiving maximally tolerated drug therapy. However, a large-scale randomized controlled trial comparing RFA with ASA or myectomy is needed to further assess the efficacy and safety of RFA [58]. The effect of $\mathrm{RF}$ on the reduction of SAM-dependant MR was not addressed well in the previously mentioned studies.

\section{Treatment of MR associated non-obstructive HCM}

Despite the fact that non-obstructive HCM have high rates of adverse clinical events, almost equivalent to those with obstructive HCM, no data available for the management of non-obstructive HCM [59]. Treatment of non-obstructive HCM associated significant MR is challenging. With the development of heart failure in non-obstructive HCM, the current pharmacological treatment does not differ from general heart failure management, except for peculiar aspects e.g. genetic counselling and testing [60]. Early referral of patients with $\mathrm{HF}$ and moderate or severe MR to a multidisciplinary Heart Team is recommended for assessment and treatment planning. Isolated MV surgery may be considered in symptomatic patients with severe MR despite optimal therapy and low surgical risk [61]. For patients with asymptomatic MV disease, earlier surgery in patients with HCM should be limited to those comprehensive HCM centers with documented evidence of the highest success rates and lowest complication rates (i.e., durable success is $>90 \%$ with an expected mortality rate $<1 \%$ ) [62].

\section{Conclusion}

In summary, MR associated HCM needs a special interest, as the optimal approach of its management is completely dependent on the better understanding of the mechanism. Integrated imaging modalities should be used to obtain detailed morphological and functional assessment of MV. Even with the availability of large 
published data and updated guidelines, the standard treatment of MR is still controversial.

\section{Financial Support}

None.

\section{References}

1. Maron MS, Olivotto I, Zenovich AG, Link MS, Pandian NG, et al. (2006) Hypertrophic cardiomyopathy is predominantly a disease of left ventricular outflow tract obstruction. Circulation 114: 2232-2239.

2. Hong JH, Schaff HV, Nishimura RA, Abel MD, Dearani JA, et al. (2016) Mitral regurgitation in patients with hypertrophic obstructive cardiomyopathy: Implications for concomitant valve procedures. J Am Coll Cardiol 68: 1497-1504.

3. Elliott PM, Anastasakis A, Borger MA, Borggrefe M, Cecchi $F$, et al. (2014) 2014 ESC guidelines on diagnosis and management of hypertrophic cardiomyopathy: The task force for the diagnosis and management of hypertrophic cardiomyopathy of the European Society of Cardiology (ESC). Eur Heart J 35: 2733-2779.

4. Sherrid MV, Balaram S, Kim B, Axel L, Swistel DG (2016) The mitral valve in obstructive hypertrophic cardiomyopathy: A test in context. J Am Coll Cardiol 67: 1846-1858.

5. Wigle ED, Sasson Z, Henderson MA, Ruddy TD, Fulop J, et al. (1985) Hypertrophic cardiomyopathy. The importance of the site and the extent of hypertrophy: A review. Prog Cardiovasc Dis 28: 1-83.

6. Jiang L, Levine RA, King ME, Weyman AE (1987) An integrated mechanism for systolic anterior motion of the mitral valve in hypertrophic cardiomyopathy based on echocardiographic observations. Am Heart J 113: 633-644.

7. Sherrid MV, Chu CK, Delia E, Mogtader A, Dwyer EM Jr (1993) An echocardiographic study of the fluid mechanics of obstruction in hypertrophic cardiomyopathy. J Am Coll Cardiol 22: 816-825.

8. Grigg LE, Wigle ED, Williams WG, Daniel LB, Rakowski H (1992) Transesophageal Doppler echocardiography in obstructive hypertrophic cardiomyopathy: Clarification of pathophysiology and importance in intraoperative decision making. J Am Coll Cardiol 20: 42-52.

9. Maron MS, Olivotto I, Harrigan C, Appelbaum E, Gibson CM, et al. (2011) Mitral valve abnormalities identified by cardiovascular magnetic resonance represent a primary phenotypic expression of hypertrophic cardiomyopathy. Circulation 124: 40-47.

10. Klues HG, Maron BJ, Dollar AL, Roberts WC (1992) Diversity of structural mitral valve alterations in hypertrophic cardiomyopathy. Circulation 85: 1651-1660.

11. Mandeş L, Roşca M, Ciupercă D, Popescu BA (2020) The role of echocardiography for diagnosis and prognostic stratification in hypertrophic cardiomyopathy. J Echocardiogr 18: 137-148.

12. Chung H, Kim Y, Park CH, Kim JY, Min PK, et al. (2019) Genetic relevance and determinants of mitral leaflet size in hypertrophic cardiomyopathy. Cardiovasc Ultrasound 17: 21.

13. Halpern DG, Swistel DG, Po JR, Joshi R, Winson G, et al. (2015) Echocardiography before and after resect-plicaterelease surgical myectomy for obstructive hyper-trophic cardiomyopathy. J Am Soc Echocardiogr 28: 1318-1328.

14. Kaple RK, Murphy RT, DiPaola LM, Houghtaling PL, Lever
$\mathrm{HM}$, et al. (2008) Mitral valve abnormalities in hypertrophic cardiomyopathy: Echocardiographic features and surgical outcomes. Ann Thorac Surg 85: 1527-1535.

15. Moro E, ten Cate FJ, Leonard JJ, Hugenholtz PG, Roelandt $J$ (1987) Genesis of systolic anterior motion of the mitral valve in hypertrophic cardiomyopathy: an anatomical or dynamic event? Eur Heart J 8: 1312-1321.

16. Oki T, Fukuda N, luchi A, Tabata T, Tanimoto $M$, et al. (1995) Transesophageal echocardiographic evaluation of mitral regurgitation in hypertrophic cardiomyopathy: Contributions of eccentric left ventricular hypertrophy and related abnormalities of the mitral complex. J Am Soc Echocardiogr 8: 503-510.

17. Petrone RK, Klues HG, Panza JA, Peterson EE, Maron BJ (1992) Coexistence of mitral valve prolapse in a consecutive group of 528 patients with hypertrophic cardiomyopathy assessed with echocardiography. J Am Coll Cardiol 20: 5561.

18. Kwon DH, Setser RM, Thamilarasan M, Popovic ZV, Smedira NG, et al. (2008) Abnormal papillary muscle morphology is independently associated with increased left ventricular outflow tract obstruction in hypertrophic cardiomyopathy. Heart 94: 1295-1301.

19. Boissier F, Achkouty G, Bruneval P, Fabiani JN, Nguyen AT, et al. (2015) Rupture of mitral valve chordae in hypertrophic cardiomyopathy. Arch Cardiovasc Dis 108: 244-249.

20. Zhu WX, Oh JK, Kopecky SL, Schaff HV, Tajik AJ (1992) Mitral regurgitation due to ruptured chordae tendinae in patients with hypertrophic obstructive cardiomyopathy. J Am Coll Cardiol 20: 242-247.

21. Silbiger JJ (2016) Abnormalities of the mitral apparatus in hypertrophic cardiomyopathy: Echocardiographic, pathophysiologic, and surgical insights. J Am SocEchocardiogr 29: 622-639.

22. Anwar AM, Soliman OI, Nemes A, Germans T, Krenning BJ, et al. (2007) Assessment of mitral annulus size and function by real-time 3-dimensional echocardiography in cardiomyopathy: Comparison with magnetic resonance imaging. J Am Soc Echocardiogr 20: 941-948.

23. Critoph $\mathrm{CH}$, Pantazis A, Tome Esteban MT, SalazarMendiguchia J, Pagourelias ED, et al. (2014) The influence of aortoseptal angulation on provocable left ventricular outflow tract obstruction in hypertrophic cardiomyopathy. Open Heart 1: e000176.

24. Spirito P, Binaco I, Poggio D, Zyrianov A, Grillo M, et al. (2019) Role of preoperative cardiovascular magnetic resonance in planning ventricular septal myectomy in patients with obstructive hypertrophic cardiomyopathy. Am J Cardiol 123: 1517-1526.

25. Turvey L, Augustine DX, Robinson S, Oxborough D, Stout M, et al. (2021) Transthoracic echocardiography of hypertrophic cardiomyopathy in adults: A practical guideline from the British Society of Echocardiography. Echo Res Pract 8: G61-G86.

26. Hang D, Schaff HV, Nishimura RA, Lahr BD, Abel $M D$, et al. (2019) Accuracy of jet direction on doppler echocardiography in identifying the etiology of mitral regurgitation in obstructive hypertrophic cardiomyopathy. $J$ Am Soc Echocardiogr 32: 333-340.

27. Cardim N, Galderisi M, Edvardsen T, Plein S, Popescu BA, et al. (2015) Role of multimodality cardiac imaging in the management of patients with hypertrophic cardiomyopathy: An expert consensus of the European Association of 
Cardiovascular Imaging Endorsed by the Saudi Heart Association. Eur Heart J Cardiovasc Imaging 16: 280.

28. Dwivedi G, Mahadevan G, Jimenez D, Frenneaux M, Steeds RP (2014) Reference values for mitral and tricuspid annular dimensions using two-dimensional echocardiography. Echo Res Pract 1: 43-50.

29. Zoghbi WA, Adams D, Bonow RO, Enriquez-Sarano M, Foster E, et al. (2017) Recommendations for noninvasive evaluation of native valvular regurgitation: A report from the American Society of Echocardiography developed in collaboration with the Society for Cardiovascular Magnetic Resonance. J Am Soc Echocardiogr 30: 303-371.

30. Śpiewak M, Kłopotowski M, Gawor M, Kubik A, Kowalik E, et al. (2017) Quantification of mitral regurgitation in patients with hypertrophic cardiomyopathy using aortic and pulmonary flow data: Impacts of left ventricular outflow tract obstruction and different left ventricular segmentation methods. J Cardiovasc Magn Reson 19: 105.

31. Song Y, Yang DH, Hartaigh BO, Cho SJ, Kang JW, et al. (2018) Geometric predictors of left ventricular outflow tract obstruction in patients with hypertrophic cardiomyopathy: A 3D computed tomography analysis. Eur Heart J Cardiovasc Imaging 19: 1149-1156.

32. Ommen SR, Mital S, Burke MA, Day SM, Deswal A, et al. (2020) 2020 AHA/ACC guideline for the diagnosis and treatment of patients with hypertrophic cardiomyopathy: A report of the American College of Cardiology/American Heart Association Joint Committee on Clinical Practice Guidelines. Circulation 142: e558-e631.

33. Olivotto I, Oreziak A, Barriales-Villa R, Abraham TP, Masri A, et al. (2020) Mavacamten for treatment of symptomatic obstructive hypertrophic cardiomyopathy (EXPLORERHCM): A randomised, double-blind, placebo-controlled, phase 3 trial. Lancet 396: 759-769.

34. Saberi S, Cardim N, Yamani M, Schulz-Menger J, Li W, et al. (2021) Mavacamten favorably impacts cardiac structure in obstructive hypertrophic cardiomyopathy: EXPLORERHCM Cardiac Magnetic Resonance Substudy Analysis. Circulation 143: 606-608.

35. Ho CY, Mealiffe ME, Bach RG, Bhattacharya M, Choudhury $\mathrm{L}$, et al. (2020) Evaluation of Mavacamten in symptomatic patients with nonobstructive hypertrophic cardiomyopathy. J Am Coll Cardiol 75: 2649-2660.

36. Maron BJ, Dearani JA, Ommen SR, Maron MS, Schaff HV, et al. (2015) Low operative mortality achieved with surgical septal myectomy: Role of dedicated hypertrophic cardiomyopathy centers in the management of dynamic subaortic obstruction. J Am Coll Cardiol 66: 1307-1308.

37. van der Lee C, Kofflard MJM, van Herwerden LA, Vletter WB, ten Cate FJ (2003) Sustained improvement after combined anterior mitral leaflet extension and myectomy in hypertrophic obstructive cardiomyopathy. Circulation 108 : 2088-2092.

38. Wei LM, Thibault DP, Rankin JS, Alkhouli M, Roberts HG, et al. (2019) Contemporary surgical management of hypertrophic cardiomyopathy in the United States. Ann Thorac Surg 107: 460-466.

39. Nampiaparampil RG, Swistel DG, Schlame M, Saric M, Sherrid MV (2018) Intraoperative two- and threedimensional transesophageal echocardiography in combined myectomy-mitral operations for hypertrophic cardiomyopathy. J Am Soc Echocardiogr 31: 275-288.

40. Collis R, Tsang V, Pantazis A, Tome-Esteban M, Elliott
PM, et al. (2018) Individualized surgical strategies for left ventricular outflow tract obstruction in hypertrophic cardiomyopathy. Eur J Cardiothorac Surg 53: 1237-1243.

41. Nasseri BA, Stamm C, Siniawski H, Kukucka M, Komoda T, et al. (2011) Combined anterior mitral valve leaflet retention plasty and septal myectomy in patients with hypertrophic obstructive cardiomyopathy. Eur J Cardiothorac Surg 40: $1515-1520$

42. Holst KA, Hanson KT, Ommen SR, Nishimura RA, Habermann EB, et al. (2019) Septal myectomy in hypertrophic cardiomyopathy: National outcomes of concomitant mitral surgery. Mayo Clin Proc 94: 66-73.

43. Balaram SK, Ross RE, Sherrid MV, Schwartz GS, Hillel Z, et al. (2012) Role of mitral valve plication in the surgical management of hypertrophic cardiomyopathy. Ann Thorac Surg 94: 1990-1997.

44. Delmo Walter EM, Javier MF, Hetzer R (2017) Long-term outcome of simultaneous septal myectomy and anterior mitral leaflet retention plasty in hypertrophic obstructive cardiomyopathy: The Berlin experience. Ann Cardiothorac Surg 6: 343-352.

45. Shah AA, Glower DD, Gaca JG (2016) Trans-aortic Alfieri stitch at the time of septal myectomy for hypertrophic obstructive cardiomyopathy. J Card Surg 31: 503-506.

46. Lapenna E, Nisi T, Ruggeri S, Trumello C, Del Forno B, et al. (2020) Edge-to-edge mitral repair associated with septal myectomy in hypertrophic obstructive cardiomyopathy. Ann Thorac Surg 110: 783-789.

47. Bogachev-Prokophiev A, Afanasyev A, Zheleznev S, Fomenko M, Sharifulin R, et al. (2017) Mitral valve repair or replacement in hypertrophic obstructive cardiomyopathy: A prospective randomized study. Interact Cardiovasc Thorac Surg 25: 356-362.

48. Shimahara Y, Fujita T, Kobayashi J, Fukushima S, Kume Y, et al. (2019) Combined mechanical mitral valve replacement and transmitral myectomy for hypertrophic obstructive cardiomyopathy treatment: An experience of over 20 years. J Cardiol 73: 318-325.

49. Rietman GW, van der Maaten JM, Douglas YL, Boonstra PW (2002) Echocardiographic diagnosis of left ventricular outflow tract obstruction after mitral valve replacement with subvalvular preservation. Eur J Cardiothorac Surg 22: 825827.

50. Kim LK, Swaminathan RV, Looser P, Minutello RM, Wong SC, et al. (2016) Hospital volume outcomes after septal myectomy and alcohol septal ablation for treatment of obstructive hypertrophic cardiomyopathy: US Nationwide Inpatient Database, 2003-2011. JAMA Cardiol 1: 324-332.

51. Liebregts $M$, Faber $L$, Jensen MK, Vriesendorp PA, Januska J, et al. (2017) Outcomes of alcohol septal ablation in younger patients with obstructive hypertrophic cardiomyopathy. JACC Cardiovasc Interv 10: 1134-1143.

52. Nguyen A, Schaff HV, Hang D, Nishimura RA, Geske JB, et al. Surgical myectomy versus alcohol septal ablation for obstructive hypertrophic cardiomyopathy: A propensity score-matched cohort. J Thorac Cardiovasc Surg 157: 306315.e3.

53. Sorajja P, Pedersen WA, Bae R, Lesser JR, Jay D, et al. (2016) First experience with percutaneous mitral valve plication as primary therapy for symptomatic obstructive hypertrophic cardiomyopathy. J Am Coll Cardiol 67: 28112818.

54. Thomas F, Rader F, Siegel RJ (2017) The use of mitraclip 
for symptomatic patients with hypertrophic obstructive cardiomyopathy. Cardiology 137: 58-61.

55. Boekstegers P, Hausleiter J, Baldus S, von Bardeleben RS, Beucher H, et al. (2014) Percutaneous interventional mitral regurgitation treatment using the Mitra-Clip system. Clin Res Cardiol 103: 85-96.

56. Poon SS, Cooper RM, Gupta D (2016) Endocardial radiofrequency septal ablation - A new option for nonsurgical septal reduction in patients with hypertrophic obstructive cardiomyopathy (HOCM)?: A systematic review of clinical studies. Int J Cardiol 222: 772-774.

57. Liu L, Li J, Zuo L, Zhang J, Zhou M, et al. (2018) Percutaneous intramyocardial septal radiofrequency ablation for hypertrophic obstructive cardiomyopathy. J Am Coll Cardiol 72: 1898-1909.

58. Yang H, Yang Y, Xue Y, Luo S (2020) Efficacy and safety of radiofrequency ablation for hypertrophic obstructive cardiomyopathy: A systematic review and meta-analysis. Clin Cardiol 43: 450-458.
59. Lu DY, Pozios I, Haileselassie B, Ventoulis I, Liu H, et al. (2018) Clinical outcomes in patients with nonobstructive, labile, and obstructive hypertrophic cardiomyopathy. J Am Heart Assoc 7: e006657.

60. McDonagh TA, Metra M, Adamo M, Gardner RS, Baumbach A, et al. (2021) 2021 ESC Guidelines for the diagnosis and treatment of acute and chronic heart failure. Eur Heart $\mathrm{J} 42$ : 3599-3726.

61. Vahanian A, Beyersdorf F, Praz F, Milojevic M, Baldus S, et al. (2021) 2021 ESC/EACTS Guidelines for the management of valvular heart disease. Eur $\mathrm{J}$ Cardiothorac Surg 60: 727-800.

62. Nishimura RA, Otto CM, Bonow RO, Carabello BA, Erwin JP 3rd, et al. (2017) 2017 AHA/ACC focused update of the 2014 AHA/ACC guideline for the management of patients with valvular heart disease: A report of the American College of Cardiology/American Heart Association Task Force on Clinical Practice Guidelines. Circulation 135: e1159-e1195. 